\title{
Relationship between psoriasis and non-alcoholic fatty liver disease
}

\author{
Krishnasamy Narayanasamy ${ }^{1}$, Abarna Devi Sanmarkan², Karthick Rajendran ${ }^{3}$, Chezhian Annasamy ${ }^{1}$, \\ Senthilkumar Ramalingam ${ }^{1}$ \\ ${ }^{1}$ Department of Hepatology, Madras Medical College, Rajiv Gandhi Government General Hospital, Chennai, India \\ ${ }^{2}$ Institute of Internal Medicine, Madras Medical College, Rajiv Gandhi Government General Hospital, Chennai, India \\ ${ }^{3}$ Multi-disciplinary Research Unit (MRU), Madras Medical College, Rajiv Gandhi Government General Hospital, Chennai, India
}

Key words: non-alcoholic fatty liver disease (NAFLD), psoriasis, metabolic syndrome.

Address for correspondence: Prof. Krishnasamy Narayanasamy, Department of Hepatology, Madras Medical College, Rajiv Gandhi Government General Hospital, 600003 Chennai, India, phone: 91-9841170145, e-mail: drkns_1963@yahoo.com

\begin{abstract}
Introduction: Various components of metabolic syndrome have an important role in the pathogenesis of both non-alcoholic fatty liver disease (NAFLD) and psoriasis, suggesting an association between these diseases. However, at present very few studies have reported on the systematic evaluations of the prevalence of NAFLD in patients with psoriasis disorder.

Aim: To investigate the prevalence of NAFLD in patients with psoriasis vulgaris. The study also evaluated the parallel relationship between both of the diseases.

Material and methods: Patients over18 years old and with a diagnosis of psoriasis vulgaris at the outpatient unit of Department of Dermatology were considered for enrolment and were followed up by the Department of Hepatology, Madras Medical College. Each and every patient completed a questionnaire, underwent a thorough skin evaluation, and had a right upper quadrant ultrasound and fasting blood workup.

Results: Two hundred and fifty patients were enrolled in the study. The participants were predominantly middle aged (mean: $44.74 \pm 11.989$ years), overweight (average body mass index (BMI): $\left.24.772 \pm 3.611 \mathrm{~kg} / \mathrm{m}^{2}\right)$, and male $(68 \%, n=170)$. The overall prevalence of NAFLD among psoriasis was $45.2 \%$.

Conclusions: Non-alcoholic fatty liver disease is highly prevalent among our cohort of patients with psoriasis, occurring in $45.2 \%$ of patients. Comorbidity of NAFLD is highly associated with psoriasis, which emphasises that both diseases may develop simultaneously. Health care providers should be mindful of this association since early evaluation and diagnosis of NAFLD in patients with psoriasis may play a vital role in alleviating the progression of liver disease.
\end{abstract}

\section{Introduction}

Psoriasis is a chronic inflammatory disease that affects the skin. Studies have shown that psoriasis is not merely a skin problem; psoriasis is linked with various comorbid conditions, especially obesity and metabolic syndrome [1-5], which are known risk factors for non-alcoholic fatty liver disease (NAFLD). In the past decade, many studies have drawn attention to comorbid conditions in psoriasis, and preliminary epidemiological data suggest that psoriasis could be associated with the development of NAFLD. Non-alcoholic fatty liver disease includes a spectrum of conditions that range from simple fatty liver, which is relatively benign, to non-alcoholic steatohepatitis (NASH), which can give rise to fibrosis, cirrhosis, and end-stage liver disease [6]. Moderate or severe conditions of psoriasis have a high prevalence of chronic liver disease [7]. It is hypothesised that components of metabolic syndrome as the most likely pathogenic basis may play a role in the manifestation of NAFLD and psoriasis.

\section{Aim \\ In the present work, our aim was to analyse the prevalence of NAFLD among psoriasis and basic aspects of the relationship between psoriasis and NAFLD.}




\section{Material and methods \\ Study population}

Patients with an age of above 18 years and with a clinical diagnosis of psoriasis vulgaris, who were outpatients at the Department of Dermatology, Madras Medical College were recruited for the study over a period of 9 months (from July 2014 to March 2015). We excluded patients who were currently on treatment and also those who received methotrexate, cyclosporine, acitretin, psoralens, or systemic steroids 30 days prior to the day of testing. Subjects who had known documented chronic liver disease (alcohol abuse, hepatitis B or C, haemochromatosis, Wilson disease, autoimmune hepatitis, primary biliary cirrhosis, primary sclerosing cholangitis or hepatic malignancy), human immunodeficiency virus (HIV), clinical evidence of malignancy, or other secondary causes of chronic liver disease were also excluded.

\section{Design of study and sample size}

This study was designed as a prospective hospital-based observational study in which 250 patients were enrolled. The sample size was determined based on the following assumptions:

1) $17.4 \%$ is the previous prevalence reported (our region),

2) $5 \%$ absolute precision,

3) $95 \%$ confidence interval.

Based on calculation, the samples required were 215 participants.

\section{Anthropometric, clinical, and laboratory variables}

Information of the study subjects including age, gender, hypertension, diabetes mellitus, dyslipidaemia, and duration of the psoriasis were collected by a standardized questionnaire. The subject's waist circumference, weight, and height were measured upon enrolment. Body mass index (BMI) was calculated as weight in kilograms divided by the square of height in metres $\left(\mathrm{kg} / \mathrm{m}^{2}\right)$. Waist circumference was measured according to WHO recommendations [8]. Hypertension or high blood pressure was diagnosed if the participant was on anti-hypertensive medications, if a physician had ever told them that they had high blood pressure, or the average of three blood pressure measurements was 130/85 mm Hg. Diabetes mellitus was diagnosed if participants were taking hypoglycaemic medications or had a fasting glucose concentration $110 \mathrm{mg} / \mathrm{dl}$ or had been told by a physician that they had diabetes mellitus. All study participants gave written informed consent, and the study protocol was approved by the Institutional Ethics Committee.
Metabolic syndrome was diagnosed by the National Cholesterol Education Program - Adult Treatment Panel III criteria (NCEP - ATP III) [9]. In accordance with the definition of metabolic syndrome, the patient was classified as having the syndrome if he/she had at least three of the following five risk abnormalities:

1) waist circumference $>102 \mathrm{~cm}$ in men or $>88 \mathrm{~cm}$ in women,

2) fasting glucose $110 \mathrm{mg} / \mathrm{dl}$ or on treatment,

3) triglycerides $\geq 150 \mathrm{mg} / \mathrm{dl}$,

4) HDL men $<40 \mathrm{mg} / \mathrm{dl}$, women $<50 \mathrm{mg} / \mathrm{dl}$ or on treatment, and

5) blood pressure $130 / 85 \mathrm{~mm} \mathrm{Hg}$ or on treatment.

Venous blood samples were drawn in the morning from each participant after an overnight fast. A panel of blood tests were performed for each participant, which included serum levels of total bilirubin, aspartate aminotransferase (AST), alanine aminotransferase (ALT), serum alkaline phosphatase (SAP), serum total protein, albumin, platelet count, viral markers (hepatitis $B$ and C infections), fasting glucose levels, total cholesterol, high density lipoprotein (HDL), and total triglycerides. Low-density lipoprotein (LDL) cholesterol was estimated by the Friedewald's equation (i.e. total cholesterol minus high-density lipoprotein cholesterol minus triglycerides divided by five).

\section{Diagnosis of psoriasis}

The diagnosis of psoriasis was made clinically by the dermatologist and disease severity was assessed using the Psoriasis Area and Severity Index (PASI) [10]. The PASI score, ranging from 0 to 72, reflects the erythema, induration, and scaling of the lesions in four body areas (head, trunk, upper limbs, and lower limbs).

\section{Diagnosis of NAFLD}

Hepatic ultrasonography scanning procedure was performed in all participants by a qualified radiologist, who was blinded to participant's particulars. The diagnosis of hepatic fatty liver was made on the basis of characteristic sonographic features, i.e. evidence of diffuse hyper-echogenicity of liver relative to kidneys, ultrasound beam attenuation, and poor visualisation of intra-hepatic structures. The fibro scan assesses the fibrosis in liver disease according to the following classification: F0 - no fibrosis, < 6.5; F1 - perisinusoidal fibrosis without septa, 6.6-8.0; F2 - portal and periportal fibrosis with rare septum, 8.1-12.5; F3 - portal and periportal fibrosis with many septa $>12.6$; and F4 - cirrhosis.

\section{Statistical analysis}

Statistical calculations were performed using of SPSS software (version 20.0). Data were expressed as 
mean \pm standard deviation for continuous variables and as numbers and percentages for categorical variables. Statistical analyses included the analysis of variance ANOVA (for continuous variables) and Pearson's $\chi^{2}$ test (for categorical variables). The association of NAFLD with the psoriasis was assessed by logistic regression analysis. Values at $p<0.05$ were considered statistically significant.

\section{Results}

\section{Baseline characteristics of the psoriasis cohort}

To evaluate the prevalence of NAFLD in people with psoriasis, we conducted 9-month hospital-based study. During the enrolment period, 340 adults with psoriasis were eligible for the study and 250 (73.52\%) agreed to participate in our study. Their characteristics are summarised in Table I. All participants were diagnosed with psoriasis vulgaris, in which some of the specific types were as follows: 49 had chronic plaque psoriasis, 45 had palmoplantar psoriasis, 38 had scalp psoriasis, 28 patients had psoriasis arthritis, 21 had pustular type, and 7 had nail type.

The participants were largely middle aged (44.74 $\pm 11.989)$ and the majority were overweight ( 24.772 $\left.\pm 3.611 \mathrm{~kg} / \mathrm{m}^{2}\right)$ and male $(68 \%, n=170)$. More than two thirds of the participants (70.8\%) met the criteria for diagnosis of metabolic syndrome based on NCEP - ATP III. Abdominal obesity was detected in $48 \%$ of psoriasis patients. Dyslipidaemia was more commonly observed in $96 \%$ of patients (abnormal value at least anyone of lipid profile), and $52.8 \%$ of patients were hyperglycaemic. $53.6 \%$ of psoriatic patients had hypertension, and $44 \%$ of the patients presented with elevated ALT levels.

\section{Prevalence and characteristics of psoriasis-associated NAFLD}

The sonographic examination revealed signs of fatty liver (134/250, 53.6\%). In 21 of these 134 cases, there was evidence of significant ethanol intake $(n=14)$, chronic hepatitis B infection (HBsAg +ve) $(n=6)$, and chronic hepatitis C (anti-HCV +ve) $(n=1)$. The remaining 113 met the criteria for diagnosis of NAFLD (prevalence: $45.2 \%)$.

Psoriasis patients with NAFLD were younger than patients with psoriasis alone, more obese when compared to non-NAFLD patients with psoriasis, more likely to be male and had higher levels of BMI. They also had greater frequency of metabolic syndrome when compared to psoriasis with non-NAFLD and the majority of NAFLD patients had higher serum ALT concentrations. Notably, compared with psoriasis patients with NAFLD had a more severe form of psoriasis than with non-NAFLD
Table I. Characteristics of psoriasis subjects $(n=250)$

\begin{tabular}{|c|c|}
\hline Characteristics & Value \\
\hline Age & $44.74 \pm 11.989$ \\
\hline Gender (male) & $170(68 \%)$ \\
\hline Weight & $62.54 \pm 9.608$ \\
\hline Height & $158.84 \pm 7.833$ \\
\hline BMI & $24.772 \pm 3.611$ \\
\hline Waist circumference & $91.53 \pm 13.910$ \\
\hline Systolic & $120.26 \pm 15.222$ \\
\hline Diastolic & $81.43 \pm 11.335$ \\
\hline FBS & $114.23 \pm 25.328$ \\
\hline TGL & $202.87 \pm 109.762$ \\
\hline Cholesterol & $214.04 \pm 40.969$ \\
\hline $\mathrm{HDL}$ & $44.28 \pm 8.572$ \\
\hline Obese & $46(18.4 \%)$ \\
\hline Metabolic syndrome & $177(70.8 \%)$ \\
\hline Fatty liver & $134(53.6 \%)$ \\
\hline NAFLD & $113(45.2 \%)$ \\
\hline Alcoholic & $22(8.8 \%)$ \\
\hline Abdominal obesity & $120(48 \%)$ \\
\hline Hypercholesterolaemia & $160(64 \%)$ \\
\hline Hypertriglyceridaemia & $166(66.4 \%)$ \\
\hline Low HDL & $117(46.8 \%)$ \\
\hline Hyperglycaemia & $132(52.8 \%)$ \\
\hline Hypertension & $134(53.6 \%)$ \\
\hline Diabetes & $133(53.2 \%)$ \\
\hline Elevated ALT & $110(44 \%)$ \\
\hline $\mathrm{HBsAg}+\mathrm{ve}$ & $9(3.6 \%)$ \\
\hline Anti-HCV +ve & $1(0.4 \%)$ \\
\hline AST & $28.15 \pm 12.009$ \\
\hline ALT & $29.75 \pm 13.005$ \\
\hline AST/ALT & $1.044 \pm 0.419$ \\
\hline Duration & $4.14 \pm 2.696$ \\
\hline Fibro Scan & $5.974 \pm 1.7435$ \\
\hline PASI & $27.80 \pm 13.494$ \\
\hline S. Bilirubin - total & $0.664 \pm 0.5997$ \\
\hline S. Bilirubin - direct & $0.304 \pm 0.1360$ \\
\hline SAP & $68.49 \pm 24.514$ \\
\hline Serum protein & $7.530 \pm 1.003$ \\
\hline Albumin & $4.239 \pm 0.4704$ \\
\hline Platelet & $2.7158 \pm 0.85809$ \\
\hline
\end{tabular}


according to PASI score (mean \pm SD: $32.88 \pm 13.542$ vs. $23.19 \pm 12.052 ; p<0.0001)$ (Table II).

Fibrosis scores of NAFLD patients with psoriasis were higher than non-NAFLD patients with psoriasis. Among the NAFLD patients with psoriasis, $36.2 \%$ showed portal and periportal fibrosis with septum (F2-F3). Most of the NAFLD patients with psoriasis (63.8\%) showed fibrosis in F1 stage. Cirrhosis of the liver did not occur in any of the NAFLD patients with psoriasis.

\section{Correlation between psoriasis with} NAFLD and psoriasis without NAFLD

Univariate logistic regression analysis was performed to explore the mechanisms underlying the cor-

Table II. Correlation between psoriasis with non-NAFLD and NAFLD

\begin{tabular}{|c|c|c|c|}
\hline Characteristics & Psoriasis with NAFLD $(n=113)$ & Psoriasis with non-NAFLD $(n=105)$ & $P$-value \\
\hline Age & $42.23 \pm 11.004$ & $46.02 \pm 12.622$ & 0.019 \\
\hline Gender (male) & $84(74.3 \%)$ & $64(61 \%)$ & 0.042 \\
\hline Weight & $63.74 \pm 9.456$ & $61.11 \pm 9.923$ & 0.046 \\
\hline Height & $158.85 \pm 7.480$ & $157.52 \pm 8.156$ & 0.212 \\
\hline BMI & $25.217 \pm 3.462$ & $24.655 \pm 3.956$ & 0.265 \\
\hline Systolic & $122.01 \pm 13.218$ & $118.93 \pm 17.791$ & 0.147 \\
\hline Diastolic & $81.56 \pm 11.721$ & $82.39 \pm 11.442$ & 0.596 \\
\hline FBS & $120.69 \pm 24.570$ & $110.70 \pm 27.273$ & 0.005 \\
\hline TGL & $238.18 \pm 129.895$ & $172.23 \pm 83.368$ & 0.0001 \\
\hline Cholesterol & $217.03 \pm 43.198$ & $210.28 \pm 40.723$ & 0.237 \\
\hline $\mathrm{HDL}$ & $44.54 \pm 8.206$ & $43.43 \pm 9.038$ & 0.342 \\
\hline LDL & $124.23 \pm 37.242$ & $132.07 \pm 34.716$ & 0.110 \\
\hline Obese & $16(14.2 \%)$ & $13(12.4 \%)$ & 0.842 \\
\hline Metabolic syndrome & $94(83.2 \%)$ & $69(65.7 \%)$ & 0.005 \\
\hline Waist circumference & $94.07 \pm 11.401$ & $88.00 \pm 15.038$ & 0.001 \\
\hline Abdominal obesity & $63(55.8 \%)$ & $39(37.1 \%)$ & 0.007 \\
\hline Hypercholesterolaemia & $69(61.1 \%)$ & $66(62.9 \%)$ & 0.889 \\
\hline Hypertriglyceridaemia & $89(78.8 \%)$ & $54(51.4 \%)$ & 0.0001 \\
\hline Low HDL & $51(45.1 \%)$ & $56(53.3 \%)$ & 0.278 \\
\hline Hyperglycaemia & $76(67.3 \%)$ & $43(41 \%)$ & 0.0001 \\
\hline Hypertension & $65(57.5 \%)$ & $59(56.2 \%)$ & 0.891 \\
\hline Diabetes & $77(68.1 \%)$ & $45(42.9 \%)$ & 0.0001 \\
\hline Elevated ALT & $66(58.4 \%)$ & $35(33.3 \%)$ & 0.0001 \\
\hline ALT & $34.21 \pm 13.666$ & $26.23 \pm 11.532$ & 0.0001 \\
\hline AST & $32.04 \pm 13.320$ & $29.30 \pm 9.617$ & 0.084 \\
\hline AST/ALT & $0.989 \pm 0.386$ & $1.258 \pm 0.500$ & 0.0001 \\
\hline Duration & $4.61 \pm 2.932$ & $3.69 \pm 2.339$ & 0.011 \\
\hline Fibro Scan & $6.596 \pm 1.852$ & $5.422 \pm 1.431$ & 0.0001 \\
\hline PASI & $32.88 \pm 13.542$ & $23.19 \pm 12.052$ & 0.0001 \\
\hline S. Bilirubin - total & $0.748 \pm 0.841$ & $0.672 \pm 0.335$ & 0.392 \\
\hline S. Bilirubin - direct & $0.341 \pm 0.152$ & $0.332 \pm 0.170$ & 0.703 \\
\hline SAP & $64.69 \pm 25.771$ & $69.87 \pm 23.355$ & 0.123 \\
\hline Serum protein & $7.635 \pm 0.849$ & $7.430 \pm 1.176$ & 0.140 \\
\hline Albumin & $4.179 \pm 0.395$ & $4.262 \pm 0.476$ & 0.160 \\
\hline Platelet & $2.647 \pm 0.540$ & $2.813 \pm 1.173$ & 0.178 \\
\hline
\end{tabular}


relation between psoriasis and NAFLD in the $218 \mathrm{pa}$ tients. The significant were observed between NAFLD and non-NAFLD subjects in age, sex, weight, waist circumference, fasting blood sugar, PASI, metabolic syndrome, ALT, duration of psoriasis, fibroscan, direct serum bilirbin, serum protein and statistical significant was not observed in other variables in univariate analysis (Table III).

Table III. Univariate analysis

\begin{tabular}{|c|c|c|}
\hline Characteristics & $P$-value & OR $(95 \% \mathrm{Cl})$ \\
\hline Age & 0.020 & $1.028(1.004-1.052)$ \\
\hline Gender (male) & 0.035 & $1.856(1.043-3.301)$ \\
\hline Weight & 0.048 & $0.972(0.945-1.0)$ \\
\hline Height & 0.212 & $0.978(0.945-1.013)$ \\
\hline BMI & 0.264 & $0.960(0.893-1.032)$ \\
\hline Systolic & 0.149 & $0.987(0.970-1.005)$ \\
\hline Diastolic & 0.595 & $1.006(0.983-1.030)$ \\
\hline FBS & 0.006 & $0.984(0.973-0.996)$ \\
\hline TGL & 0.0001 & $0.993(0.990-0.997)$ \\
\hline Cholesterol & 0.237 & $0.996(0.990-1.003)$ \\
\hline $\mathrm{HDL}$ & 0.341 & $0.985(0.955-1.016)$ \\
\hline LDL & 0.111 & 1.006 (0.999-1.014) \\
\hline Obese & 0.699 & $1.167(0.532-2.560)$ \\
\hline Metabolic syndrome & 0.004 & $2.581(1.366-4.879)$ \\
\hline Waist circumference & 0.001 & $0.966(0.947-0.987)$ \\
\hline Abdominal obesity & 0.006 & $2.132(1.239-3.669)$ \\
\hline Hypercholesterolaemia & 0.785 & $0.927(0.536-1.602)$ \\
\hline Hypertriglyceridaemia & 0.0001 & $3.510(1.939-6.327)$ \\
\hline Low HDL & 0.227 & $0.720(0.422-1.227)$ \\
\hline Hyperglycaemia & 0.0001 & $2.962(1.704-5.148)$ \\
\hline Hypertension & 0.843 & $1.056(0.617-1.805)$ \\
\hline Diabetes & 0.0001 & 2.852 (1.640-4.959) \\
\hline Elevated ALT & 0.0001 & $2.809(1.617-4.877)$ \\
\hline ALT & 0.0001 & $0.951(0.929-0.973)$ \\
\hline AST & 0.087 & $0.980(0.957-1.003)$ \\
\hline AST/ALT & 0.0001 & $4.889(2.202-10.852)$ \\
\hline Duration & 0.013 & $0.877(0.792-0.972)$ \\
\hline Fibro Scan & 0.0001 & $0.642(0.533-0.774)$ \\
\hline PASI & 0.0001 & $0.941(0.918-0.965)$ \\
\hline S. Bilirubin - total & 0.425 & $0.805(0.472-1.373)$ \\
\hline S. Bilirubin - direct & 0.702 & $0.723(0.137-3.805)$ \\
\hline SAP & 0.124 & 1.009 (0.998-1.020) \\
\hline Serum protein & 0.147 & $0.816(0.620-1.074)$ \\
\hline Albumin & 0.168 & $1.576(0.825-3.012)$ \\
\hline Platelet & 0.198 & $1.248(0.891-1.749)$ \\
\hline
\end{tabular}

Another important finding of our study is multivariate analysis, which has not been carried out in previous reports. The variables that were independently associated with NAFLD among patients with psoriasis were hypertriglyceridaemia $(\mathrm{OR}=3.174,95 \% \mathrm{Cl}: 1.565-$ $6.438, p<0.001$ ), hyperglycaemia (OR $=2.495,95 \%$ Cl: $1.191-5.227, p<0.015)$, fibro scan $(O R=0.692$, $95 \% \mathrm{Cl}: 0.553-0.867, p<0.001)$, PASI (OR = 0.950, $95 \% \mathrm{Cl}: 0.922-0.979, p<0.001)$, and duration $(\mathrm{OR}=0.865,95 \% \mathrm{Cl}: 0.756-0.990, p<0.035)$ and gender $(\mathrm{OR}=2.681,95 \% \mathrm{Cl}: 1.244-5.778, p<0.012)$ (Table IV).

\section{Discussion}

The hallmark of NAFLD refers to the accumulation of fat in the liver. Non-alcoholic fatty liver disease is regarded as the hepatic manifestation of metabolic syndrome and the most common form of chronic liver disease throughout world [11-13]. The previously well-described risk factors for NAFLD include metabolic syndrome and lifestyle habits, such as fructose consumption and physical activity level, which impact disease severity [14]. Recent reports have shown that people with psoriasis may be at greater risk for developing of NAFLD $[15,16]$.

Non-alcoholic fatty liver disease prevalence has been estimated to be in the $20-30 \%$ range in the general population in various countries and is rapidly increasing. The prevalence of NAFLD has become a major public health concern in developed and developing countries $[17,18]$. Non-alcoholic fatty liver disease is one such disease that now has potentially enormous public health consequences due to its progression to more severe forms of the disease ranging from steatosis to steatohepatitis (NASH), which, in turn, can progress into cirrhosis and end stage liver disease and finally the need for liver transplantation. The increasing incidence of NAFLD throughout world has contributed to rising numbers of HCC incidents [19]. There is also

Table IV. Multivariate analysis

\begin{tabular}{lcc} 
Characteristics & $\boldsymbol{P}$-value & AOR $(\mathbf{9 5} \% \mathrm{Cl})$ \\
\hline Age & 0.136 & $1.022(0.993-1.052)$ \\
\hline Gender (male) & 0.012 & $2.681(1.244-5.778)$ \\
\hline Weight & 0.069 & $0.967(0.933-1.003)$ \\
\hline Abdominal obesity & 0.474 & $1.291(0.642-2.593)$ \\
\hline Hypertriglyceridemia & 0.001 & $3.174(1.565-6.438)$ \\
\hline Hyperglycaemia & 0.015 & $2.495(1.191-5.227)$ \\
\hline Elevated ALT & 0.224 & $1.563(0.761-3.209)$ \\
\hline Duration & 0.035 & $0.865(0.756-0.990)$ \\
\hline Fibro Scan & 0.001 & $0.692(0.553-0.867)$ \\
\hline PASI & 0.001 & $0.950(0.922-0.979)$
\end{tabular}


evidence suggesting that NAFLD individuals are at risk of developing cardiovascular events independently of conventional risk factors and metabolic syndrome components [20].

Psoriasis is a chronic, inflammatory, immune-mediated skin disease. Both innate and adaptive immunity are crucial in the initiation and maintenance of psoriatic plaques. Non-alcoholic fatty liver disease began to be considered a part of the metabolic syndrome spectrum, and the relationship between NAFLD and psoriasis became grounded in their association with metabolic syndrome and secretion of proinflammatory cytokines. The first report of patients with psoriasis and concomitant hepatic steatosis was unexpectedly found during a survey conducted in 2002, which aimed to evaluate patients with NAFLD. These patients presented with liver abnormalities seen on ultrasonography and underwent a liver biopsy, which revealed hepatic fibrosis. Since then, different population-based surveys have shown a higher prevalence of NAFLD in patients with psoriasis compared with the general population. It was also observed that the PASI was higher in patients with both psoriasis and NAFLD than in those with psoriasis only.

We have conducted a study to determine the prevalence of NAFLD among patients with psoriasis in our region. The present study reports a notable increase in the prevalence of NAFLD when compared with a previous study in 2012 carried out in our region, in which $17 \%$ prevalence was reported [21]. Our study is the first to confirm that patients with psoriasis are at increased risk for NAFLD, as suggested by previous studies that used the same evaluating measures for fatty liver; however, our study showed that the prevalence of NAFLD was $45.2 \%$, i.e. the prevalence rate of NAFLD is more than 2.5 -fold higher. It should be noted that we do not feel the results of our study discredit the possibility of NAFLD prevalence among psoriasis as determined in other studies conducted in Italy, Netherland, and the USA, which demonstrated that the prevalence was $46 \%, 47 \%$, and $47 \%$, respectively [22-24].

Many studies showed that elderly patients are at increased risk of NAFLD with psoriasis even if the skin disease is mild or moderate and not requiring systemic therapy $[24,25]$. But an important finding of our study showed that a high prevalence is observed even in middle-aged psoriatic populations; the mean age of patients was 44.02 years.

We observed a significant relationship between NAFLD and gender. NAFLD was more prevalent in male gender than in female gender. High BMI levels were frequent in our NAFLD patients with psoriasis, and this finding confirmed the previous findings [21].
It is reported that metabolic syndrome is more common in psoriasis patients [26]. We found higher rates of metabolic syndrome among NAFLD than non-NAFLD patients with psoriasis. However, our major finding in this study was that psoriasis with NAFLD was positively correlated with three components of metabolic syndrome: hyperglycaemia, hypertriglyceridemia, and abdominal obesity, but significant correlation was not observed with hypertension and low level of HDL in univariate logistic regression analysis, although the levels of $\mathrm{HDL}$ and blood pressure were reported to be higher in psoriasis patients with NAFLD than in those with non-NAFLD.

Although obesity is reported to be highly prevalent among psoriasis patients, we did not find significant correlation between BMI and NAFLD in psoriasis patients. However, we observed a higher number of obese among psoriasis with NAFLD patients than non-NAFLD psoriasis patients. This may be explained by which concluded that the predominant of our psoriasis patients were overweight.

In our study we observed that ALT was increased in psoriasis patients with NAFLD and highly associated with psoriasis. We did not detect any significant association of NAFLD in other liver function tests in our psoriasis patients. Our study found that DM was common in psoriasis patients. We also found that hypercholesterolaemia was not associated with NAFLD patients with psoriasis even though TGL was highly significantly associated with NAFLD patients with psoriasis.

In multivariate analysis, NAFLD was associated with both the severity and duration of psoriasis. We also observed that psoriasis is associated with hyperglycaemia and hypertriglyceridemia. Therefore, it was concluded that NAFLD is related to the duration of the disease, components of metabolic syndrome such hyperglycaemia and hypertriglyceridemia, and closely associated with the development of psoriasis and NAFLD.

\section{Conclusions}

Our findings suggest that NAFLD is frequent in patients with psoriasis and is also associated with the duration and severity of the disease. Physicians must be careful of this association and evaluation of NAFLD in patients with psoriasis, but future investigation is required to determine the most appropriate diagnostic and treatment strategies for these patients.

\section{Conflict of interest}

The authors declare no conflict of interest.

\section{References}

1. Davidovici BB, Sattar N, Prinz JC, et al. Psoriasis and systemic inflammatory diseases: potential mechanistic links between 
skin disease and co-morbid conditions. J Invest Dermato 2010; 130: 1785-96.

2. Azfar RS, Gelfand JM. Psoriasis and metabolic disease: epidemiology and pathophysiology. Curr Opin Rheumatol 2008; 20 : 416-22.

3. Boehncke WH, Boehncke S, Schon MP. Managing comorbid disease in patients with psoriasis. BMJ 2010; 340: b5666.

4. Menter A, Griffiths CE, Tebbey PW, et al. Exploring the association between cardiovascular and other disease-related risk factors in the psoriasis population: the need for increased understanding across the medical community. J Eur Acad Dermatol Venereol 2010; 24: 1371-7.

5. Paschos P, Paletas K. Non alcoholic fatty liver disease and metabolic syndrome. Hippokratia 2009; 13: 9-19.

6. Adams LA, Lymp JF, Sauver J, et al. The natural history of nonalcoholic fatty liver disease: a population-based cohort study. Gastroenterology 2005; 129: 113-21.

7. Vilarrasa E, Puig L. Psoriasis: biologic treatment and liver dis ease. World J Dermatol 2014; 3: 76-85.

8. Waist Circumference and Waist-Hip Ratio, Report of a WHO Expert Consultation, Geneva, 8-11 December 2008.

9. Expert Panel on Detection, Evaluation, and Treatment of High Blood Cholesterol in Adults. Executive summary of the third report of The National Cholesterol Education Program (NCEP) expert panel on detection, evaluation, and treatment of high blood cholesterol in adults (Adult Treatment Panel III). JAMA 2001; 285: 2486-97.

10. Smith CH, Anstey AV, Barker JN, et al. British Association of Dermatologists guidelines for use of biological interventions in psoriasis 2005. Br J Dermatol 2005; 153: 486-97.

11. Paschos P, Paletas K. Non alcoholic fatty liver disease and metabolic syndrome. Hippokratia 2009; 13: 9-19.

12. Anand SS, Yusuf S, Vuksan V, S, et al. Differences in risk factors, atherosclerosis, and cardiovascular disease between ethnic groups in Canada: the Study of Health Assessment and Risk in Ethnic Groups (SHARE). Lancet 2000; 356: 279-84.

13. Younossi ZM, Stepanova M, Negro F, et al. Nonalcoholic fatty liver disease in lean individuals in the United States. Medicine (Baltimore) 2012; 91: 319-27.

14. Abdelmalek MF, Suzuki A, Guy C, et al. Increased fructose consumption is associated with fibrosis severity in patients with nonalcoholic fatty liver disease. Hepatology 2010; 51: 1961-71.

15. Miele L, Vallone S, Cefalo C, et al. Prevalence, characteristics and severity of non-alcoholic fatty liver disease in patients with chronic plaque psoriasis. J Hepatol 2009; 51: 778-86.

16. Gisondi P, Targher G, Zoppini G, et al. Non-alcoholic fatty liver disease in patients with chronic plaque psoriasis. J Hepato 2009; 51: 758-64

17. Hossain P, Kawar B, El Nahas M. Obesity and diabetes in the developing world - a growing challenge. N Engl J Med 2007 356: 213-5.

18. Das K, Mukherjee PS, Ghosh A, et al. Non obese population in a developing country has a high prevalence of non-alcoholic fatty liver and significant liver disease. Hepatology 2010; 51: 1593-602.

19. Kikuchi L, Oliveira CP, Carrilho FJ. Nonalcoholic fatty liver disease and hepatocellular carcinoma. Biomed Res Intern 2014 2014: 106247
20. Ballestri S, Lonardo A, Bonapace S, et al. Risk of cardiovascular, cardiac and arrhythmic complications in patients with non-alcoholic fatty liver disease World J Gastroenterol 2014; 20: 1724-45.

21. Madanagobalane S, Anandan S. The increased prevalence of non-alcoholic fatty liver disease in psoriatic patients: a study from South India. Australas J Dermatol 2012; 53: 190-7.

22. Van der Voort EA, Koehler EM, Dowlatshahi EA, et al. Psoriasis is independently associated with nonalcoholic fatty liver disease in patients 55 years old or older: results from a population-based study. J Am Acad Dermatol 2014; 70: 517-24.

23. Gisondi P, Targher G, Zoppini G, Girolomoni G. Non-alcoholic fatty liver disease in patients with chronic plaque psoriasis. J Hepatol 2009; 51: 758-64.

24. Roberts KK, Cochet AE, Lamb PB, et al. The prevalence of NAFLD and NASH among patients with psoriasis in a tertiary care dermatology and rheumatology clinic. Aliment Pharmacol Ther 2015; 41: 293-300.

25. Tseng HW, Lin HS, Lam HC. Co-morbidities in psoriasis: a hospital-based case-control study. J Eur Acad Dermatol Venereol 2013; 27: 1417-25.

26. Gisondi P, Tessari G, Conti A, et al. Prevalence of metabolic syndrome in patients with psoriasis: a hospital-based case-control study. Br J Dermatol 2007; 157: 68-73.

Received: 17.04 .2015

Accepted: 10.07.2015 